\title{
The anti-evaporative mechanism of wax esters - Implications for the function of tear fluid
}

\author{
Riku O. Paananen, Antti H. Rantamäki and Juha M. Holopainen* \\ Helsinki Eye Lab, Department of Ophthalmology, University of Helsinki, Helsinki, Finland
}

Funding: The Finnish Eye Foundation and the Sigrid Juselius Foundation supported this study.

\footnotetext{
*Corresponding author: Juha Holopainen, Helsinki Eye Lab, Department of Ophthalmology, University of Helsinki, P.O. Box 220, 00029 HUS, Finland; E-mail: juha.holopainen@hus.fi; Tel.: +358-9-471 77197; Fax $+358-9-47175100$
} 


\section{Supporting information}

\section{BAM calibration}

According to the Fresnel equations, the reflectance for p-polarized light at an interface of two materials with refractive indices $n_{1}$ and $n_{2}$ is

$$
R_{\mathrm{p}}=\left|\frac{n_{1} \sqrt{1-\left(\frac{n_{1}}{n_{2}} \sin \theta_{\mathrm{i}}\right)^{2}}-n_{2} \cos \theta_{\mathrm{i}}}{n_{1} \sqrt{1-\left(\frac{n_{1}}{n_{2}} \sin \theta_{\mathrm{i}}\right)^{2}}+n_{2} \cos \theta_{\mathrm{i}}}\right|^{2}
$$

where $\theta_{\mathrm{i}}$ is the angle if incidence. The refractive index of the aqueous subphase can be varied by adding different concentrations of $\mathrm{NaCl}^{1}{ }^{1}$ Reflected intensity per unit time was measured from the surface of water containing $0,9,20,30,50,60$ and $90 \mathrm{~g} / \mathrm{L} \mathrm{NaCl}$. Intensity per unit time was used instead of average intensity in order to obtain intensity measurements from a wider range of reflectance values without working near the sensitivity limit of the detector or having overexposed pixels in the images. Reflectance at different concentrations of $\mathrm{NaCl}$ was calculated using eq $\mathrm{A} 1$ to create the calibration curve shown in Figure S1. A power function was fitted to the data and used to obtain reflectance from intensity-per-unittime data in further measurements.

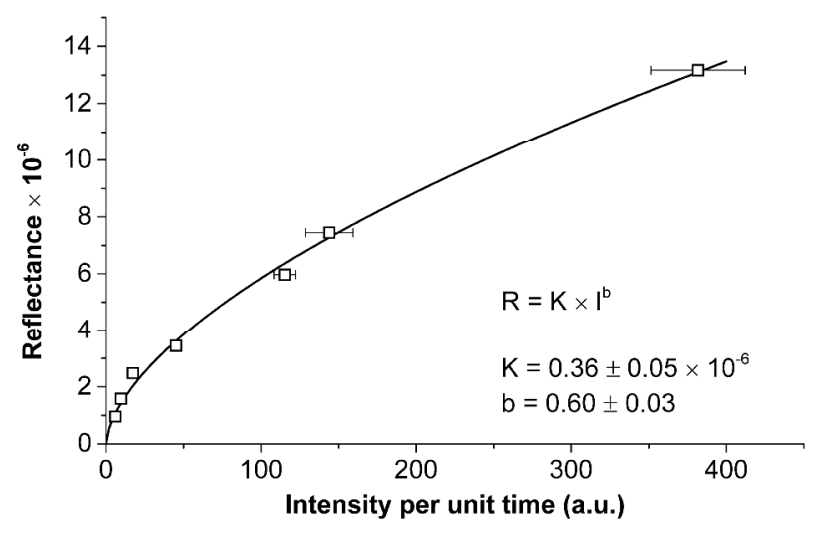

Figure S1. Calibration curve relating the intensity per unit time (I, mean \pm SE) measured from BAM images to the reflectance $(R)$ of the film. 
1-Octadecanol, 1-Docosanol, 1-Hexacosanol and 1-Triacontanol were added to the air-water interface of a Langmuir trough in $5 \mathrm{mM}$ chloroform solution and chloroform was allowed to evaporate for 10 minutes. Trough temperature was maintained within $36 \pm 1{ }^{\circ} \mathrm{C}$ during the experiments. Films were compressed to a mean molecular area of $18 \AA^{2}$ at a speed of $1.2\left(\AA^{2} /\right.$ molecule $) / \mathrm{min}$. Average image intensity per unit time was measured from all of the films and intensity values were converted into reflectance using the fit shown in Fig. S1. A theoretical thickness for the linear alcohols in all-trans conformation was calculated according to Tanford. ${ }^{2}$ A linear fit (Fig. S2) was used to obtain values of $11.6 \pm 0.4 \times 10^{3} \AA$ (value \pm SE) for $A$ and $1.3 \pm 0.2 \times 10^{-6}$ for $R_{\mathrm{bg}}$ (eq 2 ).

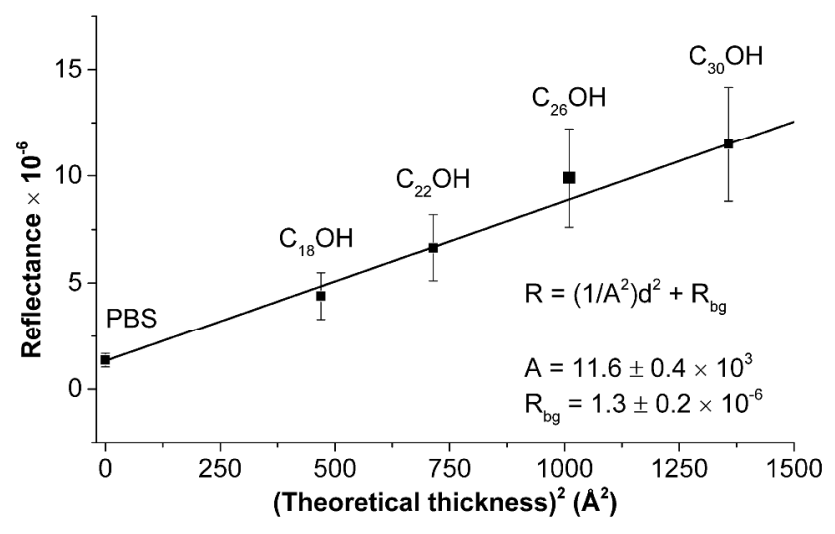

Figure S2. Fit between theoretical film thickness (d) and measured reflectance (R, mean \pm SE) of linear alcohol monolayers with chain lengths of $18,22,26$ and 30 carbons at a mean molecular area of $18 \AA^{2}$.

\section{Humidity calibration}

In non-vacuum conditions, evaporation rate $J$ from an open water surface can be expressed by ${ }^{3}$

$$
J=\frac{C_{\mathrm{s}}^{\mathrm{sat}}\left(T_{\mathrm{s}}\right)-C_{\infty}\left(T_{\infty}\right)}{R_{\mathrm{k}}+R_{\mathrm{e}}}
$$

where $C_{\mathrm{s}}^{\mathrm{sat}}$ is the water vapor concentration in equilibrium with the liquid phase and $C_{\infty}$ is the water vapor concentration far from the surface. $T_{\mathrm{s}}$ is the temperature of the water surface and $T_{\infty}$ is the temperature far from the surface. $R_{\mathrm{k}}$ is the evaporation resistance for the kinetic escape of the water molecules from the 
liquid water into the thin vapor layer adjacent to the surface and $R_{\mathrm{e}}$ is the evaporation resistance for mass transfer by diffusion and convection from the vapor layer to the surrounding atmosphere.

The resistance for kinetic escape from pure water surface is negligible, ${ }^{3}$ therefore evaporation rate from pure water surface is

$$
J_{\mathrm{w}}=\frac{C_{\mathrm{s}}^{\mathrm{sat}}\left(T_{\mathrm{s}}\right)-C_{\infty}\left(T_{\infty}\right)}{R_{\mathrm{e}}}=-\frac{C_{\infty}^{\mathrm{sat}}\left(T_{\infty}\right)}{R_{\mathrm{e}}} H_{\mathrm{R}}+\frac{C_{\mathrm{s}}^{\mathrm{sat}}\left(T_{\mathrm{s}}\right)}{R_{\mathrm{e}}}
$$

where $H_{\mathrm{R}}$ is relative humidity in the air above the film and $C_{\infty}^{\text {sat }}$ is the saturated water vapor concentration at $T_{\infty}$. Temperature far from the water surface remained constant at $22 \pm 1{ }^{\circ} \mathrm{C}$ during the experiments independent of the measurement temperature. At this temperature, $C_{\infty}^{\text {sat }}$ is $19 \pm 2 \mathrm{~g} / \mathrm{m}^{3}$. Temperature variation also has very little effect on $R_{\mathrm{e}} \cdot{ }^{3}$ In order to estimate $R_{\mathrm{e}}$ and the effect of humidity on the evaporation rate, all the control measurements at $35^{\circ} \mathrm{C}$ and $37{ }^{\circ} \mathrm{C}$ were pooled and a least squares method was used to simultaneously fit two linear functions to the data (Figure S3). Same slope was required for both fits but separate intercepts were allowed, since $C_{\mathrm{s}}^{\text {sat }}$ varies with measurement temperature.

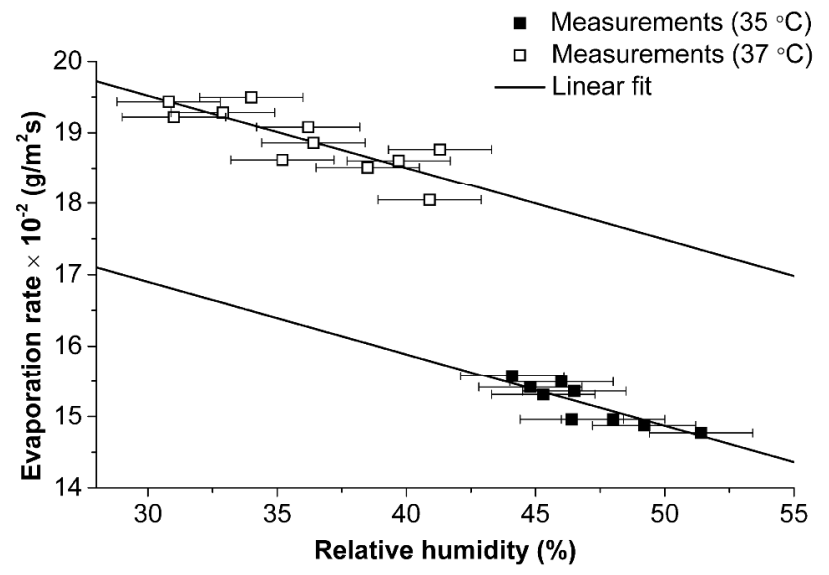

Figure S3. Evaporation rate from the surface of pure PBS buffer at $35^{\circ} \mathrm{C}$ and $37^{\circ} \mathrm{C}$. Error bars depict the average humidity variation during the experiment. 
From the linear fit, a value of $1.9 \pm 0.3 \mathrm{~s} / \mathrm{cm}$ was determined for the mass transfer resistance $R_{\mathrm{e}}$. According to the intercepts, water vapor concentration at the surface is $43 \pm 6 \mathrm{~g} / \mathrm{m}^{3}$ at $37{ }^{\circ} \mathrm{C}$ and $38 \pm 5 \mathrm{~g} / \mathrm{m}^{3}$ at $35^{\circ} \mathrm{C}$, corresponding to the saturated water vapor concentration at these temperatures.

\section{Derivation of equation 7}

In a monolayer film where solid and fluid phases coexist and mean molecular area of both phases is constant, the surface fraction covered by the solid phase can be expressed by

$$
\Phi=\frac{a_{2}\left(a-a_{1}\right)}{a\left(a_{2}-a_{1}\right)}
$$

where $a_{1}$ and $a_{2}$ are the mean molecular areas of the fluid and solid phases and $a$ is the total mean molecular area.

A monolayer residing at the air-water interface is considered to increase the resistance for the kinetic escape from water surface, $R_{\mathrm{k}}$ (see eq. A2). Assuming that the evaporation rates through a solid and a fluid monolayer of lipid are constant, the total evaporation resistance can be expressed as a function of condensed phase coverage as

$$
R_{\mathrm{k}}=\left[\Phi \frac{1}{R_{\mathrm{k}, 2}}+(1-\Phi) \frac{1}{R_{\mathrm{k}, 1}}\right]^{-1}
$$

where $R_{\mathrm{k}, 1}$ and $R_{\mathrm{k}, 2}$ are the evaporation resistances of the fluid and solid layer, respectively. When conditions between experiments are constant, evaporation reduction $E$ can be defined as

$$
E=1-\frac{J}{J_{\mathrm{w}}}=1-\frac{R_{\mathrm{e}}}{R_{\mathrm{k}}+R_{\mathrm{e}}}
$$

By combining equations $\mathrm{A} 5$ and $\mathrm{A} 6, E$ can be expressed as a function of surface coverage 


$$
E=\left[\Phi \frac{R_{\mathrm{e}}}{R_{\mathrm{k}, 2}}+(1-\Phi) \frac{R_{\mathrm{e}}}{R_{\mathrm{k}, 1}}+1\right]^{-1}
$$

Evaporation resistance of the fluid $(\Phi=0)$ and solid $(\Phi=1)$ film can be calculated from the beginning and plateau of the evaporation reduction curve (Fig. 3) by

$$
R_{\mathrm{k}, 2}=\frac{R_{\mathrm{e}} E_{\mathrm{f}}}{1-E_{\mathrm{f}}} \text { and } R_{\mathrm{k}, 1}=\frac{R_{\mathrm{e}} E_{0}}{1-E_{0}}
$$

where $E_{\mathrm{f}}$ is the evaporation reduction caused by a solid monolayer completely covering the surface and $E_{0}$ is the evaporation reduction caused by a fluid monolayer completely covering the surface.

By combining equations A4 and A7, the total evaporation reduction can be expressed as a function of the total mean molecular area as

$$
E=\left[\frac{a_{2}\left(a-a_{1}\right)}{a\left(a_{2}-a_{1}\right)}\left(\frac{R_{\mathrm{e}}}{R_{\mathrm{k}, 2}}-\frac{R_{\mathrm{e}}}{R_{\mathrm{k}, 1}}\right)+\frac{R_{\mathrm{e}}}{R_{\mathrm{k}, 1}}+1\right]^{-1}
$$

(eq 7 in the article). 


\section{References}

1. Grange, B. W.; Stevenson, W. H.; Viskanta, R. Refractive index of liquid solutions at low temperatures: an accurate measurement. Appl. Opt. 1976, 15, 858-859.

2. Tanford, C. The Hydrophobic Effect: Formation of Micelles and Biological Membranes, 2nd ed.; J. Wiley.: New York, 1980.

3. Cerretani, C. F.; Ho, N. H.; Radke, C. J. Water-evaporation reduction by duplex films: application to the human tear film. Adv. Colloid Interface Sci. 2013, 197-198, 33-57. 